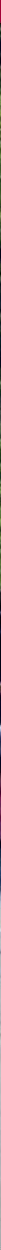

Unfallforschung kompakt

\title{
Verkehrstechnische Auswirkungen der Sonderphase für Linksabbieger an Knotenpunkten mit Lichtsignalanlage
}

Unfallforschung der Versicherer 
Impressum

Gesamtverband der Deutschen Versicherungswirtschaft e. V.

Unfallforschung der Versicherer

Wilhelmstraße 43 / 43G, 10117 Berlin

Postfach 0802 64, 10002 Berlin

unfallforschung@gdv.de

www.udv.de

Redaktion: Dipl.-Ing. Jörg Ortlepp

Layout: Michaela Gaebel

Technik: Monika Kratzer-Butenhof

Bildnachweis: Unfallforschung der Versicherer

Erschienen: 10/2010 


\section{Vorbemerkung}

An lichtsignalgesteuerten Knotenpunkten ereignen sich sowohl innerorts als auch außerorts immer wieder schwerste Unfälle, wenn Linksabbieger mit dem entgegenkommenden Verkehr oder dem parallel freigegebenen Fußgänger- bzw. Radverkehr kollidieren. Nach Berechnungen der UDV halbieren sich die Unfallkosten an Landstraßenknotenpunkten, wenn eine separate Phase für Linksabbieger eingerichtet wird. Dagegen gibt es bei den Entscheidungsträgern jedoch häufig Widerstand, weil angeblich der Verkehrsfluss verlangsamt wird. Die UDV hat deshalb in einer Auftragsstudie durch die Technische Universität Dresden ermitteln lassen, ob dieses Argument zutrifft. Die Simulation unterschiedlichster Kreuzungsformen und Ampelphasen ergab, dass sich durch eine zusätzliche gesicherte Grünphase für Linksabbieger in der Regel keine relevanten Einschränkungen der Leistungsfähigkeit der Kreuzung ergeben. 


\section{Inhalt}

$\begin{array}{ll}\text { Vorbemerkung } & 2\end{array}$

$1 \quad$ Einleitung und Ziel 4

$2 \quad$ Linksabbieger an lichtsignalgeregelten Knotenpunkten 5

2.1 Verträgliche und nichtverträgliche Ströme 5

2.2 Linksabbieger als nichtverträgliche Ströme 5

2.3 Linksabbieger als bedingt verträgliche Ströme 5

2.4 Auswahl von Untersuchungsfällen 6

2.5 Ausgangsbedingungen für die Berechnungen 6

2.6 Ergebnisse der Simulationsrechnungen 7

2.6.1 Große Kreuzung mit Dreiecksinseln (Fall 6) 7

2.6.2 Große Kreuzung ohne Dreiecksinseln (Fall 4) 10

2.6.3 Geometrisch mittlere Kreuzung (Fall 3) 12

2.6.4 Kleine Kreuzung mit je einem Fahrstreifen in allen Zufahrten (Fall 1) 14

2.6.5 Kombination ungleicher Knotenpunktzufahrten

2.6.6 Gesicherte Freigabe des Linksabbiegers als Vorgabezeit, Zugabezeit oder kombiniert, jeweils mit fester und variabler Länge $\quad 15$

2.6.7 Auswirkungen unterschiedlicher Umlaufzeiten 16

2.6.8 Mögliche Verkehrsstärken von Linksabbiegern bei hoher Belastung im Gegenverkehr 16

2.6.9 Gesamtkapazitäten von Knotenpunkten mit
unterschiedlicher Steuerungsform

$3 \quad$ Einsatzempfehlungen 18

$4 \quad$ Fazit 19

$\begin{array}{lr}\text { Literatur } & 20\end{array}$ 


\section{Einleitung und Ziel}

Knotenpunkte im Straßennetz können unterschiedliche bauliche und betriebliche Formen haben. Die Kombination von baulicher Form und Verkehrsregelung wird als Knotenpunkttyp (Eckstein/Meewes 2002) bzw. Knotenpunktart (Entwurf der Richtlinie für die Anlage von Landstraßen RAL) bezeichnet.

Bei höherer Verkehrsbelastung werden in aller Regel vorfahrtgeregelte Knotenpunkte von lichtsignalgesteuerten Knotenpunkttypen abgelöst. Durch eine Lichtsignalanlage (LSA) wird der Verkehrsraum innerhalb eines Knotenpunktes in zyklischer Folge (Phasenfolge) abwechselnd den konfligierenden Verkehrsströmen zur Verfügung gestellt. Der Knotenpunkt wird dann von den Verkehrsströmen nacheinander passiert. Soweit nicht eine planfreie Lösung oder ein Kreisverkehrsplatz infrage kommt, gelten lichtsignalgeregelte Kreuzungen und Einmündungen als Knotenpunkttypen mit hoher Leistungsfähigkeit und guter Verkehrssicherheit. Allerdings haben Untersuchungen deutliche Differenzierungen dieser Auffassung nachgewiesen: Eine Lichtsignalsteuerung macht eine Kreuzung nicht in jedem Fall sicherer als eine Vorfahrtregelung, da je nach Art der Steuerung und nach Anzahl der Phasen zunächst nur eine Veränderung in der Struktur der Sicherheitsdefizite auftreten kann, aber keine Verbesserung der Verkehrssicherheit.

Nach den Richtlinien für Lichtsignalanlagen (RiLSA 2010) werden LSA zur „Verbesserung der Verkehrssicherheit und der Qualität des Verkehrsablaufs eingerichtet. [...] Die Einrichtung einer Lichtsignalanlage ist sinnvoll, wenn Unfälle zu erwarten sind oder sich ereignet haben, die durch eine Lichtsignalsteuerung hätten vermieden werden können, und wenn sich andere Maßnahmen (z. B. Geschwindigkeitsbeschränkungen, Überholverbote, bauliche Querungsanlagen für Radfahrer und Fußgänger) als wirkungslos erwiesen haben oder keinen Erfolg versprechen." Dabei sind Lichtsignalanlagen an Kreuzungen und Einmündungen für den Fahrverkehr nach $\S 37$ der allgemeinen Verwaltungsvorschrift zur Straßenverkehrsordnung (VwV-StVO) u. a. dann erforderlich, wo es wegen fehlender Übersicht immer wieder zu Unfällen kommt und es nicht möglich ist, die Sichtverhältnisse zu verbessern oder den kreuzenden oder einmündenden Verkehr zu verbieten und wo immer wieder die Vorfahrt verletzt wird, ohne das dies mit schlechter Erkennbarkeit der Kreuzung oder mangelnder Verständlichkeit der Vorfahrtregelung zusammenhängt. Gemäß den VwV-StVO, zu den $\S \S$ 39 bis 43 gilt zudem der Grundsatz: „Die Flüssigkeit des Verkehrs ist mit den zur Verfügung stehenden Mitteln zu erhalten. Dabei geht die Verkehrssicherheit aller Verkehrsteilnehmer der Flüssigkeit des Verkehrs vor."

In der Untersuchung sollte deshalb geklärt werden, welche verkehrstechnischen Auswirkungen unterschiedliche Lichtsignalregelungen zum Schutz von Linksabbiegern haben. Insbesondere sollte dargestellt werden, inwieweit behauptete Nachteile im Verkehrsablauf tatsächlich auftreten und wie sie minimiert bzw. durch die Verbesserung in der Verkehrssicherheit kompensiert werden können.

Die Untersuchung wurde von der Technischen Universität Dresden, Fakultät Verkehrswissenschaften, Fachgebiet Straßenverkehrstechnik, unter Leitung von Prof Dr. Reinhold Maier durchgeführt. 


\section{Linksabbieger an lichtsignal- geregelten Knotenpunkten}

\subsection{Verträgliche und nichtver- trägliche Ströme}

„Die einzelnen Entwurfskomponenten der StraBenverkehrsanlage wie z. B. die Einteilung von Knotenpunktzufahrten in Fahrstreifen, die Führung der Fußgänger und Radfahrer und die Signalisierung der einzelnen Verkehrsströme sind so aufeinander abzustimmen, dass bei allen vorkommenden Belastungen und Betriebsbedingungen ein sicherer Verkehrsablauf gewährleistet wird." (s. RiLSA 2010, Kap. 1.1). Linksabbieger an lichtsignalgeregelten Knotenpunkten können unterschiedlich abgewickelt werden. Dabei sind Ströme, die keine gemeinsame Konfliktfläche besitzen als verträgliche Ströme anzusehen und können somit gemeinsam in einer Phase freigegeben werden. Ergeben sich für einzelne Verkehrsströme eine gemeinsame Konfliktfläche, so werden diese als nicht verträgliche Verkehrsströme bezeichnet. Ihre Freigabe erfolgt voneinander getrennt. Eine Ausnahme stellen dabei die nicht gesondert signalisierten Abbieger dar, die in den RiLSA als bedingt verträgliche Ströme bezeichnet werden.

\subsection{Linksabbieger als nichtver- trägliche Ströme}

Da Linksabbieger sowohl mit dem Gegenverkehr als auch mit Fußgängern und Radfahrern in gleicher Richtung gemeinsame Konfliktflächen haben, gelten sie vom Grundsatz als nichtverträgliche Ströme und müssen in unterschiedlichen Phasen freigegeben werden. Sie gelten dann als signaltechnisch gesichert geführt, wenn während ihrer Freigabe alle mit innen konfligierenden Ströme gesperrt sind. Wird so verfahren, entstehen mehrphasige Steuerungen, bei denen nacheinander die einzelnen Geradeausverkehre und Abbiegeverkehre freigegeben werden.

\subsection{Linksabbieger als bedingt verträgliche Ströme}

Während verträgliche Ströme in einer Phase zusammengefasst werden dürfen, müssen nichtverträgliche Verkehrsströme generell getrennt signalisiert werden. Eine Ausnahme gilt für abbiegende Verkehrsströme: Diese dürfen signaltechnisch nicht gesichert gleichzeitig mit Geradeausströmen geführt werden. Für sie gelten die Vorrangregeln nach $\S 9$ Abs. 3 und 4 StVO. Solche nicht gesondert signalisierten Abbiegeströme werden in den Richtlinien als bedingt verträgliche Ströme bezeichnet. Diese Abbiegeströme müssen den Vorrang des parallelen (Fußgänger- und Radverkehrs-)Stromes beachten und den entgegenkommenden Verkehrsstrom „durchsetzen“, d. h., es ist die Entscheidung der einzelnen Verkehrsteilnehmer, welche Zeitlücken sie im übergeordneten Strom annehmen oder ablehnen.

Wird die Freigabe für Linksabbiegeströme in einen "gesicherten“ und einen „ungesicherten" Teil untergliedert, wird dies als zeitweilig gesicherte Freigabe bezeichnet. Dabei wird nach Vorgabezeiten und Zugabezeiten unterschieden, die per Definition eine Zeitdauer darstellen, um die für einen oder mehrerer Verkehrsströme die Freigabezeit eher beginnt/ später endet als für andere in gleicher Phase freigegebene Verkehrsströme (RiLSA 2010). Diese Zeiten können grundsätzlich mit und ohne Anzeige durch ein Hilfssignal gesendet werden. Die Anzeige erfolgt durch einen Grünpfeil für die gesicherte Freigabezeit und ggfs. ein gelb blinkendes Signal für die Freigabezeit mit Durchsetzen. Vorgabezeiten ohne Anzeige gelten als nicht vertretbar aus Sicherheitsgründen, Zugabezeiten ohne Anzeige sind nicht empfehlenswert, da die Zeiten schlecht ausgenutzt werden. 


\subsection{Auswahl von Untersuchungs- fällen}

Folgende Kriterien wurden für die zu betrachtenden Untersuchungsfälle angelegt:

- Knotenpunkte mit einstreifiger Führung des Geradeausverkehrs in allen Richtungen.

- Kein Einfluss parallel geführter nicht motorisierter Verkehrsteilnehmer. Die Berücksichtigung von Fußgängern und Radfahrern würde sich bei nicht gesicherter Freigabe negativ auf Verkehrsqualität auswirken ${ }^{11}$.

- Keine deutliche Längsneigung, keine ungewöhnlichen Fahrstreifenbreiten und keine besonders großen oder besonders kleinen Abbiegeradien.

\subsection{Ausgangsbedingungen für die Berechnungen}

Für den Vergleich der unterschiedlichen Steuerungsvarianten sind Simulationsläufe durchgeführt worden. Für die Simulationen wurden folgende Eingangsgrößen aus den aktuellen Regelwerken und neueren Untersuchungsergebnissen verwendet:

- Umlaufzeit $\mathrm{t}_{\mathrm{u}}=60 \mathrm{~s}$,

- Zwischenzeitenberechnung nach RiLSA 1992²),

- Geschwindigkeiten in der Zufahrt $v=70 \mathrm{~km} / \mathrm{h}$ und somit Gelbzeiten von $5 \mathrm{~s}$,

- keine Gelbfahrt, wenn sich das Fahrzeug bei Gelbbeginn mehr als $10 \mathrm{~m}$ vor der Haltlinie befindet,

- Sättigungsverkehrsstärken für den Geradeausfahrer von $q_{s}=2000 \mathrm{Fz} / \mathrm{h}$, für den Linksabbieger von $q_{\mathrm{s}}=1950 \mathrm{Fz} / \mathrm{h}\left(\mathrm{t}_{\mathrm{F}}>10 \mathrm{~s}\right) \mathrm{bzw}$. $q_{\mathrm{s}}=1900 \mathrm{Fz} / \mathrm{h}\left(\mathrm{t}_{\mathrm{F}}:\right.$ 6-10 s) und für den Rechtsabbieger von $q_{s}=1800 \mathrm{Fz} / \mathrm{h}, q_{\mathrm{s}}=1700 \mathrm{Fz} / \mathrm{h}$ für zeitweilig gesicherte Linksabbieger,
- Grenz- bzw. Folgezeitlücken für durchsetzende Linksabbieger von 5,9 s bzw. 2,5 s ohne Rechtsabbiegeeinrichtung und $6,4 \mathrm{~s}$ bzw. 3,0 s mit Rechtsabbiegeeinrichtung,

- keine Schwerlastfahrzeuge, nur reine PkwStröme und

- keine nicht motorisierten Verkehrsteilnehmer.

Die unterschiedlichen Knotenpunktformen wurden mit verschiedenen Steuerungsvarianten simuliert. Jede Signalisierungsvariante wurde mit sechs verschiedenen Startzufallszahlen simuliert.

Bei der festen Freigabezeit wurden - ausgehend vom maximalen Zufluss in allen Fahrstreifen (1000 Fz/(h*Fahrstreifen)) - die Verkehrsstärken der übergeordneten Rechtsabbieger und Geradeausfahrer schrittweise verringert. Somit ergeben sich größere Zeitlücken, die bei ausreichender Größe vom Linksabbieger genutzt werden können. Zuerst wurde die maximale Verkehrsstärke ohne Linksabbiegeeinfluss bestimmt (Grundknotenkapazität) und im weiteren Verfahren mit den Anteilswerten 0,8 - 0,6 - 0,5 - 0,4 - 0,3 - 0,2 - 0,1 - 0,0 verkleinert. Der Fall „ohne Geradeausfahrer und Rechtsabbieger" dient zur Bestimmung (theoretisch) der größtmöglichsten Verkehrsstärke für den Linksabbieger.

Bei der variablen Freigabezeit sind die Verkehrsstärken der Geradeausfahrer und Rechtsabbieger nicht verändert worden. Um in jedem Umlauf einen gesättigten Zustand zu erhalten, sind die Verkehrsstärken der zufließenden Verkehrsströme für alle Fahrbeziehungen mit 1000 Fz/h je Fahrstreifen gewählt worden. Zuerst ist von einer Mindestfreigabezeit für den

1) Bei gesichertem Linksabbiegen durch eigene Grünphase tritt kein parallel freigegebener Fußgänger- und Radverkehr auf. Demgegenüber wirken sich die Fußgänger-/Radverkehrsströme bei ungesichertem Linksabbiegen je nach Verkehrsstärke negativ auf die Leistungsfähigkeit aus. Die Ergebnisse wären dadurch zugunsten der gesicherten Abbiegephasen beeinflusst worden. Um die Vergleichbarkeit der beiden Signalisierungsformen jedoch sicherzustellen und auch Betriebszeiten und Knotenpunktformen mit keinem oder sehr geringem Fußgänger-/Radverkehr abzubilden, wurde auf den Einfluss des nichtmotorisierten Verkehrs verzichtet.

2) Unter Berücksichtigung der praktischen Umsetzung, bei der die Freigabezeit nicht vor Ende der Gelbzeit beginnt. Zum Zeitpunkt der Untersuchung war noch die alte Richtlinie von 1992 gültig. 
Linksabbieger von $t_{F}=5 \mathrm{~s}$ ausgegangen worden, so dass sich für die Geradeausfahrer und Rechtsabbieger eine maximale Freigabezeit ergibt. Anschließend wurde die Freigabezeit für den Linksabbieger schrittweise um $2 \mathrm{~s}$ bis zum Maximum erhöht. Die Freigabezeiten für den übergeordneten Gegenverkehr bewegen sich immer vom Maximum bis zur minimalen Freigabezeit nach RiLSA von $t_{F, \text { min }}=5 \mathrm{~s}$. Die Fälle „keine Linksabbieger“ bzw. „keine Rechtsabbieger/Geradeausfahrer" sind zur Bestimmung der theoretischen Werte maximaler Abflussmöglichkeiten verwendet worden.

\subsection{Ergebnisse der Simulations- rechnungen}

\subsubsection{Große Kreuzung mit Dreiecksinseln (Fall 6)}

Betrachtet wird eine Kreuzung, die in allen Zufahrten drei Fahrstreifen aufweist, der Rechtsabbieger fließt frei neben der Dreiecksinsel ab. Für den Fall der signaltechnischen Sicherung des Linksabbiegers ist dieser Rechtsabbiege- strom allerdings signaltechnisch ebenfalls gesichert (Ausnahme: „Diagonalpfeil“ mit Vorgabezeit oder Zugabezeit).

Die Simulationen gehen aufgrund der Größe der Kreuzung von einer Umlaufzeit $t_{u}=90 \mathrm{~s}$ aus. Bei einer Vollauslastung aller Knotenpunktzufahrten ergibt sich für den Geradeaus-/Rechtsabbiegeverkehr eine Freigabezeit $t_{F}=39 \mathrm{~s}$ und bedeutet eine maximale Verkehrsstärke des Geradeausverkehrs von $815 \mathrm{Kfz} / \mathrm{h}$ (der freie Rechtsabbieger fließt unabhängig davon ab). Abbildung 1 zeigt diese Verkehrsstärke des Gegenverkehrs (Geradeausfahrer) in Abhängigkeit der Zunahme der Linksabbiegebelastung für unterschiedliche Phasensysteme und Regelungsformen des Linksabbiegers.

Im Fall der einfachen Zweiphasenregelung findet der Linksabbieger keine eigene gesicherte Zeit vor. Linksabbieger können daher bevorzugt während des Phasenwechsels abfließen. Bei höchster Auslastung im Gegenverkehr können daher je Umlauf bis zu drei Fahrzeuge

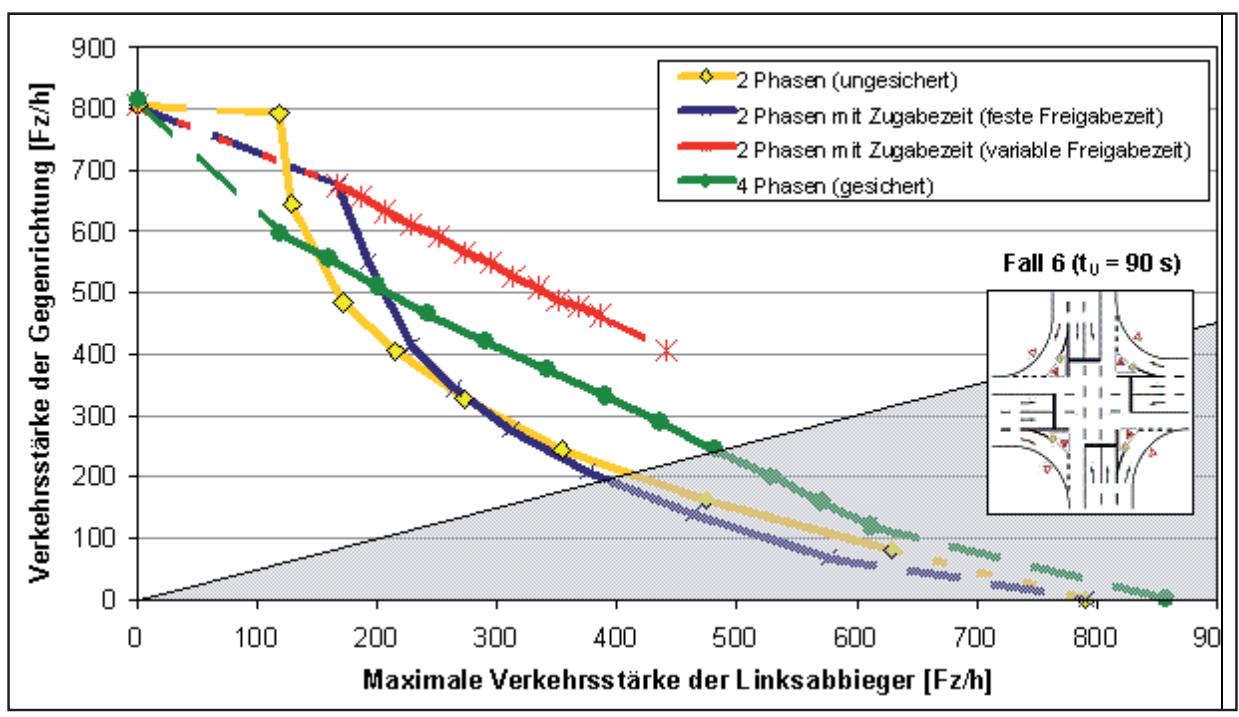

\section{Abbildung 1:}

Vergleich des Einflusses eines konfligierenden Linksabbiegers bei unterschiedlichen Steuerungsverfahren für den Linksabbiegeverkehr auf die maximale Verkehrsstärke der übergeordneten Geradeausfahrer für große Kreuzungen mit Dreiecksinseln für eine Umlaufzeit von $90 \mathrm{~s}$ 
abfließen, die sich aufgrund der geometrischen Größe des Knotenpunktes im Knoteninnenbereich aufgestellt haben. Dies führt zu einer maximalen Verkehrsstärke der Linksabbieger von ca. $120 \mathrm{Fz} / \mathrm{h}$ bei gleichzeitig maximaler Verkehrsstärke der Gegenrichtung. Eine deutliche Erhöhung der Verkehrsstärke der Linksabbieger ist erst dann zu beobachten, wenn die Verkehrsstärke der Gegenrichtung unter 400 bis $500 \mathrm{Fz} / \mathrm{h}$ fällt: Bei 400 Fahrzeugen in der Gegenrichtung können ca. 215 Fahrzeuge als Linksabbieger abfließen, d. h., ca. $100 \mathrm{Fz} / \mathrm{h}$ finden ausreichend große Lücken im Gegenverkehr. Geht die Verkehrsstärke des Geradeausverkehrs noch deutlicher zurück, so können mehr Linksabbieger abfließen. Wenn allerdings die Verkehrsstärke der Linksabbieger stärker wird als diejenige der Geradeaus-/ Gegenverkehrsströme, hierbei sind die im Fall 6 frei abfließenden Rechtsabbieger ebenfalls zu berücksichtigen, findet in der Regel eine Veränderung der Knotenpunktgeometrie oder der Regelungsart statt, da dann die Linksabbieger die dominierenden Ströme werden und z. B. mehr als einen Fahrstreifen erhalten oder der Knotenpunkt umgebaut wird. Deshalb werden die im schraffierten Bereich dargestellten Kurvenverläufe als nicht bedeutsam betrachtet, vor allem nicht für ungesicherte Linksabbiegefreigabe.

Wird in einer 2-Phasensteuerung eine Vorgabezeit oder Zugabezeit eingerichtet, so fehlt diese Zeit für den Gegenverkehr im Umlauf. Dies führt zu einem Rückgang der Verkehrsstärke des Gegenverkehrs bei gleichzeitiger Zunahme der Verkehrsstärke der Linksabbieger. Die Ergebnisse bei Vor- und Zugabezeit unterscheiden sich nur geringfügig. Wird die zusätzliche Freigabezeit für den Linksabbieger fest begrenzt, so tritt der Abbruch der Gegenverkehrsmöglichkeiten bei einer Verkehrsstärke von ca. $700 \mathrm{Fz} / \mathrm{h}$ auf, da die Freigabezeit auf $28 \mathrm{~s}$ reduziert werden muss.
Bei variabler Zugabe-/Vorgabezeit verlängern sich die Verläufe zugunsten der Verkehrsstärke der Linksabbieger wie in Abbildung 1 zu sehen. Die Ergebnisse bei einer Vorgabezeit sind bezüglich der Leistungsfähigkeit jedenfalls etwas günstiger als bei einer Zugabezeit, da selbst bei Erscheinen des diagonalen Grünpfeils Linksabbieger nicht unverzüglich fahren, während bei einer Vorgabezeit der Start der Linksabbieger unmittelbar mit Freigabezeitbeginn erfolgt.

Im Fall der gesicherten Freigabe in allen Zufahrten (4-Phasensteuerung) wird unterstellt, dass die Phase für Linksabbieger verkehrsabhängig geschaltet wird. Bei dem untersuchten hoch ausgelasteten Knotenpunkt wird die Phase sehr häufig in Anspruch genommen werden. Falls bei geringeren Anforderungen der Linksabbieger nur einzelne Linksabbiegephasen angefordert werden, ergeben sich Zwischenwerte unterhalb 120 Linksabbieger/h bis zur Vollausnutzung der Freigabezeiten im Geradeausverkehr bei nahezu keinem Linksabbiegeverkehr. In der Realität dürften diese Fälle selten auftreten, da aus Sicherheitsgründen regelmäßig Sonderphasen für den Linksabbieger eingeschaltet werden, auch wenn am Detektor keine Anmeldung vorliegt: Es kann nicht ausgeschlossen werden, dass Fahrzeuge vom Sensor nicht erkannt worden sind. Je häufiger Linksabbiegephasen angefordert werden und diesen Anforderungen auch stattgegeben wird, desto stärker geht die Verkehrsstärke des Gegenverkehrs aufgrund veränderter Freigabezeiten zurück. Der Unterschied in der Leistungsfähigkeit insgesamt zeigt aber, dass die gesicherte Freigabe der Linksabbieger mit einer 4-Phasensteuerung bei Verkehrsstärken von deutlich mehr als $200 \mathrm{Fz} / \mathrm{h}$ als Linksabbieger mehr Gegenverkehrsfahrzeuge zulässt als bei ungesichertem Durchsetzen des Linksabbiegers.

Insgesamt lassen sich im Vergleich der Steuerungsvarianten folgende Eckwerte erkennen: 
- Bis zu ca. 120 Linksabbiegern/h ist durch das Abfließen dieser Fahrzeuge während des Phasenwechsels ein Abbiegen ohne Sonderphase und damit ohne Einschränkung des Gegenverkehrs möglich.

- Bereits bei nur geringer Zunahme der Linksabbieger (z.B. 200 linksabbiegende Fahrzeuge/h), hat die gesicherte Freigabe mit einer 4-Phasensteuerung deutliche Leistungsfähigkeitsvorteile für die Gegenrichtung.

- Das Maximum des Vorsprungs für die gesicherte Freigabe liegt bei etwa ausgewogenen Belastungsverhältnissen zwischen Gegenverkehr und Linksabbiegern im Bereich um $350 \mathrm{Fz} / \mathrm{h}$.

- Von der Leistungsfähigkeit her günstiger ist die gesicherte Freigabe des Linksabbiegers in einer Zugabezeit, wenn diese variabel nach Bedarf geschaltet wird. Allerdings kann diese Freigabezeit nicht beliebig verlängert werden, da für die Gegenrichtung im Geradeausverkehr eine Mindestgrünzeit reserviert bleibt und auch in der Gegenrichtung Linksabbieger auftreten können, die ebenfalls eine Freigabezeit erhalten sollen. Daher endet der Verlauf dieser Verkehrsstärkelinien mit variabler Freigabezeit im mittleren Bereich der Verkehrsbelastung.
Diese Betrachtung schließt ausschließlich den Linksabbiegestrom und seinen gegengerichteten Geradeausstrom ein. Wegen der Dreiecksinseln bleibt der Rechtsabbieger in der Gegenrichtung außer Betracht. Wie sich diese Schaltungen bei gleichen Voraussetzungen auf die Gesamtleistungsfähigkeit des Knotenpunktes auswirken zeigt Abbildung 2. Dort ist abzulesen, welche Gesamtverkehrsstärke ein Knotenpunkt mit Lichtsignalanlage abwickeln kann, wenn bestimmte Anteile dieser Gesamtbelastung durch Linksabbieger verursacht werden und verschiedene Steuerungssystem installiert sind. Im Einzelnen lässt Abbildung 2 Folgendes erkennen:

- Die Gesamtbelastung des hier untersuchten Knotenpunktes liegt maximal bei knapp 7000 $\mathrm{Fz} / \mathrm{h}$. Dies wird erreicht, wenn zwei Phasen eingerichtet sind, der Linksabbiegeanteil aber nicht mehr als $7 \%$ beträgt und diese Linksabbieger vollständig während des Phasenwechsels abfließen können.

- Bei gesicherter Freigabe der Linksabbieger mit einer 4-Phasensteuerung vermindert sich die Gesamtleistungsfähigkeit auf etwas mehr als $6000 \mathrm{Fz} / \mathrm{h}$ wegen der notwendigen

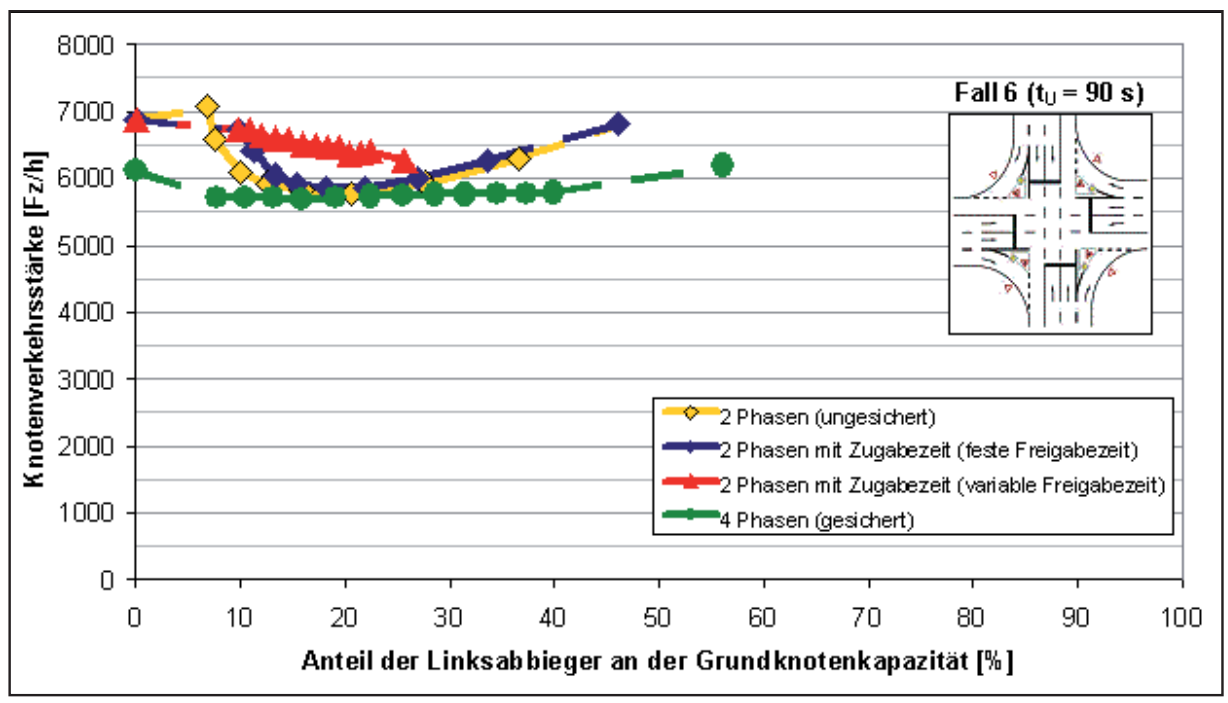

Abbildung 2:

Knotenverkehrsstärke bei verschiedenen Steuerungsformen für große Kreuzungen mit Dreiecksinseln (Fall 6) für eine Umlaufzeit von $90 \mathrm{~s}$ 
Zwischenzeiten und der Notwendigkeit, die Rechtsabbieger auch neben den Dreiecksinseln zeitweise zu sperren (Eine gesicherte Linksabbiegephase darf nur geschaltet werden, wenn kein feindlicher Strom gleichzeitig freigegeben ist). Diese Abminderung könnte $u$. $U$. vermieden werden, wenn die Dreiecksinsel derart aufgeweitet wird, dass der freie Rechtsabbieger nicht mehr als Wartepflichtiger in den Knotenpunkt einfließt, sondern anschließend in Form einer Verflechtungsstrecke in die durchgehende Fahrbahn zugeführt wird.

- Bei zunehmenden Linksabbiegeanteilen zwischen $7 \%$ bis ca. $18 \%$ nähern sich die Leistungsfähigkeiten der unterschiedlichen Regelungsformen an und erreichen zwischen 20 und $25 \%$ vergleichbare Werte, die bei etwa $5800 \mathrm{Fz} / \mathrm{h}$ liegen.

- Bei weiter zunehmenden Linksabbiegeanteilen gewinnt in der Gesamtleistungsfähigkeit eine Regelung ohne Linksabbiegeschutz deshalb, weil die Rechtsabbieger dort frei fließen können.

- Die anscheinend hohe Leistungsfähigkeit von Regelungen mit Zugabezeit, vor allem wenn diese variabel gestaltet ist, gilt nur unter der Voraussetzung, dass die freien Rechtsabbieger ohne Signalanlage abfließen können. Aus Sicherheitsgründen sowie aus Leistungsfähigkeitsgründen setzt dies aber die bereits erwähnte geometrisch aufwändige Lösung voraus, bei der mit Verflechtungsfahrstreifen diese Rechtsabbieger problemlos in den Hauptstrom eingefädelt werden können.

Zusammengefasst bedeutet dies, dass die unter diesen geometrischen Randbedingungen um ca. 20 \% höhere Leistungsfähigkeit des Knotenpunktes dadurch zustande kommt, dass bei gesicherter Freigabe der Linksabbieger die gegenüberliegenden Rechtsabbiegeströme zeitweise gesperrt werden müssen. Allerdings kommt dieser Vorteil nur zum Tragen, wenn der Linksabbiegeanteil deutlich unter $10 \%$ oder 30
$\%$ und mehr an der Gesamtbelastung beträgt. In häufig auftretenden Belastungsverhältnissen sind die Gesamtleistungsfähigkeiten nur unwesentlich verschieden.

\subsubsection{Große Kreuzung ohne Dreiecksinseln (Fall 4)}

Untersucht wurde eine große Kreuzung ohne Dreiecksinseln und mit einer Umlaufzeit von $90 \mathrm{~s}$, um den Vergleich zum Fall 6 (entsprechende Geometrie mit Dreiecksinseln) herstellen zu können. Außerdem wird dieser Fall auch mit $60 \mathrm{~s}$ Umlaufzeit berechnet. Dies dient auch der Vergleichbarkeit mit kleinen Knotenpunkten mit nur einem Fahrstreifen je Zufahrt, die tendenziell mit kleinen Umlaufzeiten betrieben werden.

Bei $90 \mathrm{~s}$ Umlaufzeit ergibt sich das in Abbildung 3 gezeigte Bild bezüglich der Verkehrsstärken auf der Konfliktfläche Linksabbieger/Gegenverkehr. Der Gegenverkehr besteht in diesem Fall aus Geradeaus- und Rechtsabbiegeverkehr.

Im Unterschied zur Kreuzung mit Dreiecksinseln beträgt die Verkehrsstärke der Gegenrichtung knapp $1600 \mathrm{Fz} / \mathrm{h}$, da zwei Fahrstreifen vorhanden sind. Eine geringe Abminderung der doppelten Verkehrsstärke bei Fall 6 kommt aus geänderten Zwischenzeiten.

Der Verlauf der Verkehrsstärkenrückgänge bei unterschiedlichen Phasensystemen ist qualitativ ähnlich wie im Fall 6 (mit Dreiecksinseln), ist allerdings in der quantitativen Auswirkung erheblich größer: Die Leistungsfähigkeitsrückgänge der ungesicherten Freigabe sind erheblich, da der Gegenverkehr in zwei Fahrstreifen ankommt. Dadurch ist die Wahrscheinlichkeit ausreichend großer Zeitlücken für das Durchsetzen der Linksabbieger erst dann in ausreichendem Maße gegeben, wenn die Verkehrsstärke in der Gegenrichtung sehr klein wird (weniger als $500 \mathrm{Fz} / \mathrm{h}$ ). Bei der gesicherten Freigabe im 4-Pha- 


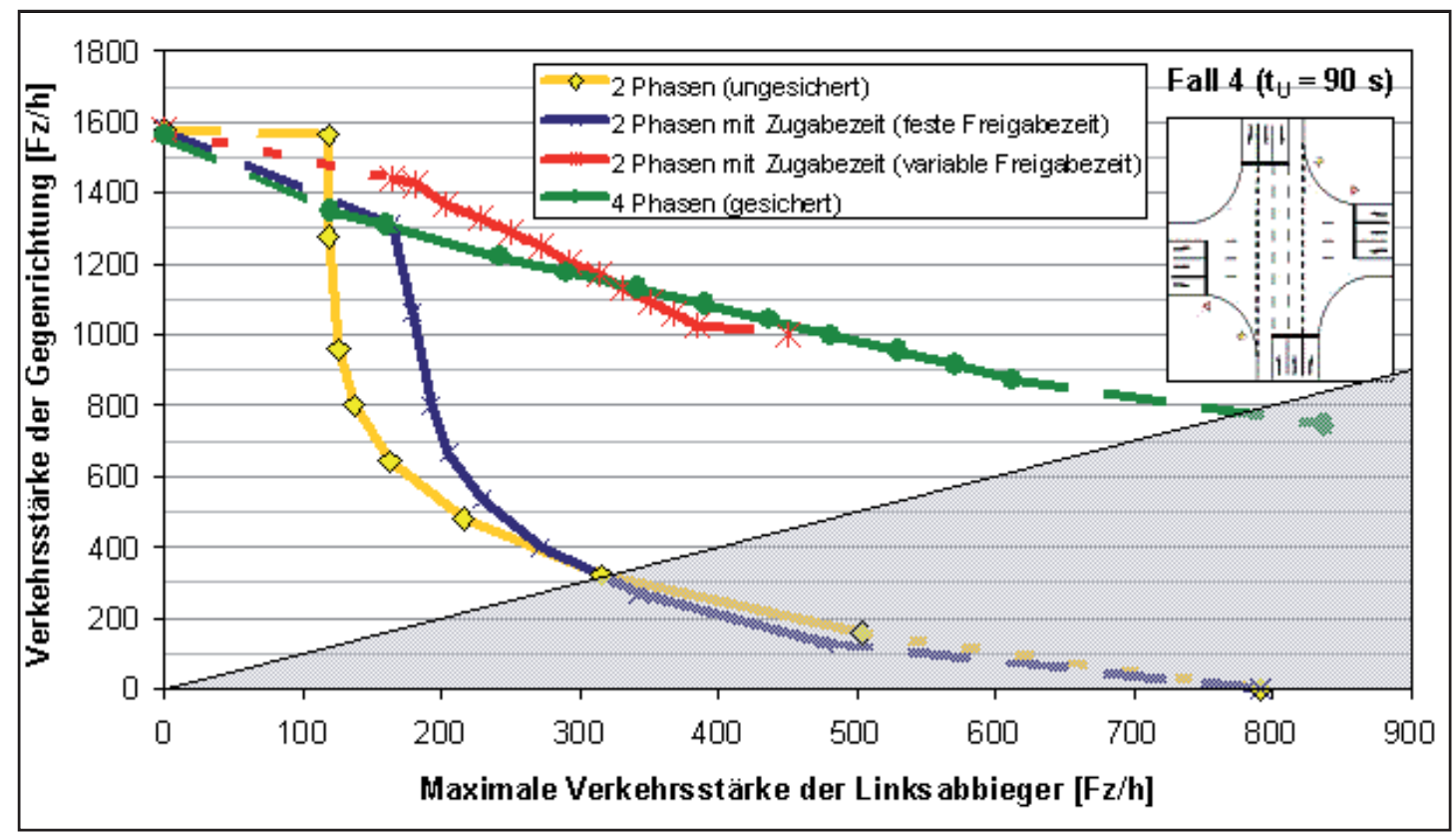

Abbildung 3:

Vergleich des Einflusses eines konfligierenden Linksabbiegers bei unterschiedlichen Steuerungsverfahren für den Linksabbiegeverkehr auf die maximale Verkehrsstärke der übergeordneten Gegenrichtung für große Kreuzungen ohne Dreiecksinseln (Fall 4) für eine Umlaufzeit von 90 s

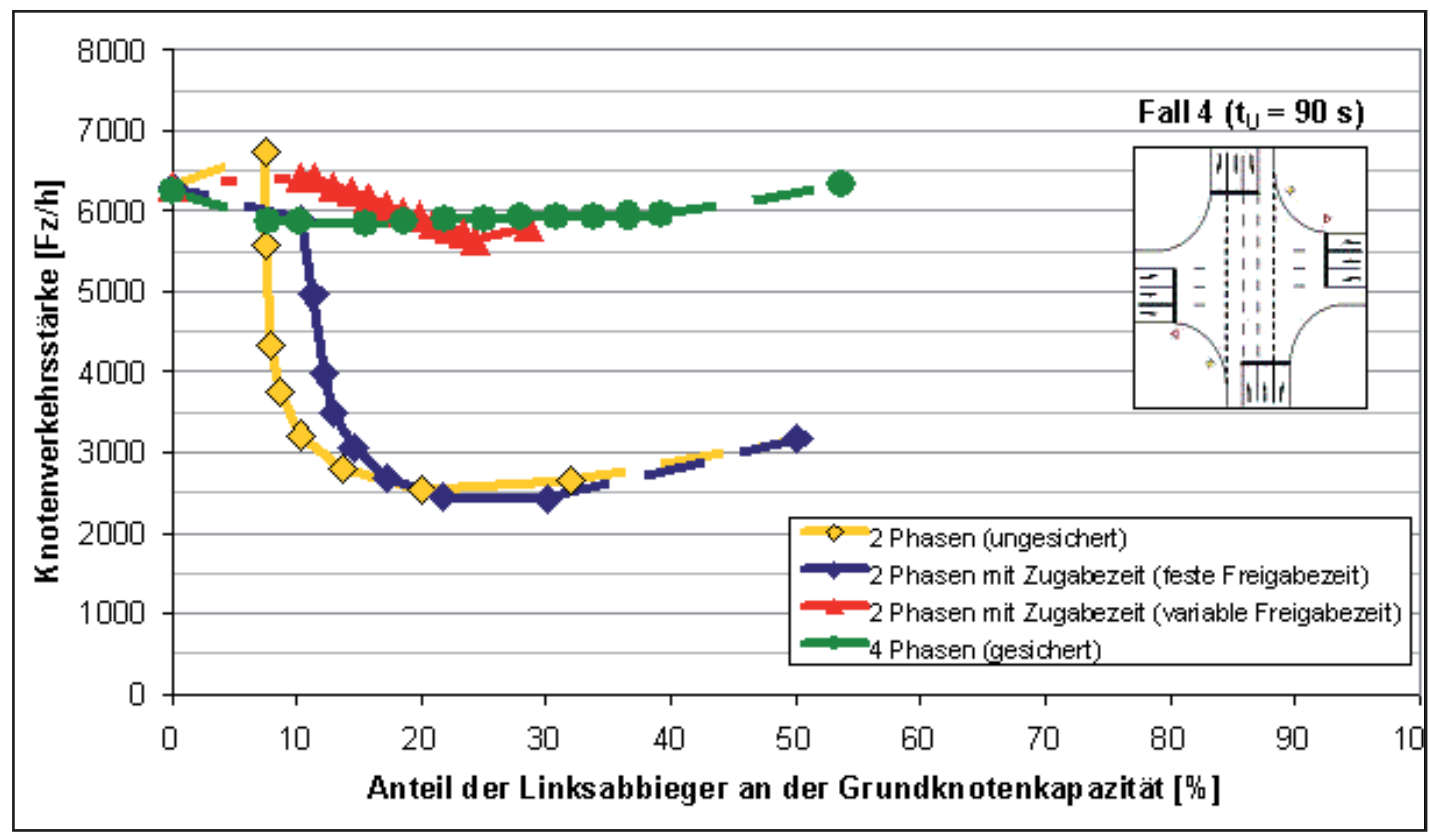

Abbildung 4:

Knotenverkehrsstärke bei verschiedenen Steuerungsformen für große Kreuzungen ohne Dreiecksinseln (Fall 4) für eine Umlaufzeit von $90 \mathrm{~s}$ 
sensystem sind dagegen bei hoher Linksabbiegebelastung Verkehrsstärken zwischen 800 und $1000 \mathrm{Fz} / \mathrm{h}$ problemlos abzuwickeln. Dies kommt auch daher, dass gleichzeitig Rechtsabbieger parallel zum gegengerichteten Linksabbiegeverkehr freigegeben werden können.

Die Leistungsfähigkeit des gesamten Knotenpunktes in Abhängigkeit des Linksabbiegeanteils zeigt Abbildung 4. Folgende Ergebnisse sind dieser Abbildung zu entnehmen:

- Die Gesamtverkehrsstärke liegt bei maximal $6300 \mathrm{Fz} / \mathrm{h}$.

- Der Rückgang der Gesamtknotenkapazität für den Fall nicht gesicherter Linksabbieger ist erheblich.

- Bei gesicherten Linksabbiegeströmen mit einer 4-Phasensteuerung bleibt dagegen die Gesamtkapazität des Knotenpunktes im Bereich um $6000 \mathrm{Fz} / \mathrm{h}$ unabhängig vom Linksabbiegeanteil.

Im Fall von mehr als einem Fahrstreifen des Gegenverkehrs, der hier untersucht wurde, ist daher die gesicherte Führung des Linksabbiegers nicht nur aus Sicherheitsgründen dringend erforderlich, sondern auch aus Leistungsfähigkeitsüberlegungen unbedingt zu empfehlen.

Bei einer Umlaufzeit von 60 s reduziert sich die Gesamtkapazität des Knotenpunktes geringfügig auf $6000 \mathrm{Fz} / \mathrm{h}$. Die Leistungsfähigkeit ist jedoch wegen des größeren Zwischenzeitenanteils geringer.

\subsubsection{Geometrisch mittlere Kreuzung (Fall 3)}

Die hier untersuchte Kreuzung zeichnet sich durch einen vergleichsweise häufig vorkommenden Fall mit zwei Fahrstreifen je Zufahrt aus. Der rechte Fahrstreifen ist jeweils für Geradeaus- und Rechtsabbiegeverkehr, der linke Fahrstreifen für den Linksabbiegever- kehr vorgesehen. Die Berechnung erfolgt mit einer Umlaufzeit von 90 s. Eine gesonderte Sicherung oder Freigabe für Rechtsabbieger ist nicht möglich wegen der gemeinsamen Nutzung mit dem Geradeausverkehr, so dass bei gesicherter Linksabbiegefreigabe der gesamte Gegenverkehr gesperrt werden muss.

Die maximalen Verkehrsstärken an der Konfliktfläche des Linksabbiegers mit dem Gegenverkehr zeigt Abbildung 5. Es ist zu erkennen, dass bei geringer Linksabbiegebelastung die Gegenrichtung bis zu ca. $830 \mathrm{Fz} / \mathrm{h}$ abfließen kann. Bei mehr als 120 Linksabbiegern/h ist die Möglichkeit des Linksabbiegeabflusses während des Phasenwechsels überschritten und es sind weitere Linksabbiegemöglichkeiten nur gegeben, wenn große Zeitlücken im Gegenstrom auftreten, so dass die Verkehrsstärke des Gegenverkehrs deutlich absinken muss, z. B. auf weniger als $400 \mathrm{Fz} / \mathrm{h}$. Bei diesen Belastungen sind regelmäßig die Leistungsfähigkeit des Gegenverkehrs bei einer 4-Phasensteuerung oder bei variabel gestalteter Zugabezeit wesentlich günstiger.

Die Gesamtkapazität des Knotenpunktes in Abhängigkeit des Linksabbiegeanteils ist in Abbildung 6 dargestellt. Hier zeigt sich sehr deutlich der Vorteil der gesicherten Freigabe bei größeren Linksabbiegeanteilen in der Gesamtkapazität:

- Bis zu 14 \% Linksabbiegeanteil hat zwar das Zweiphasensystem eine deutlich größere Leistungsfähigkeit als alle anderen Steuerungsarten. Bei üblicherweise zu erwartenden Linksabbiegeanteilen von 20 bis $40 \%$ ist aber diese Steuerungsform besonders ungünstig.

- Die gesicherte Freigabe für die Linksabbieger (4-Phasensteuerung) führt zu einer Gesamtkapazität des Knotenpunktes von ca. 3000 Fz/h (was in etwa der Hälfte der Kapazität von mehrstreifigen Knotenpunkten im Fall 4 entspricht). 


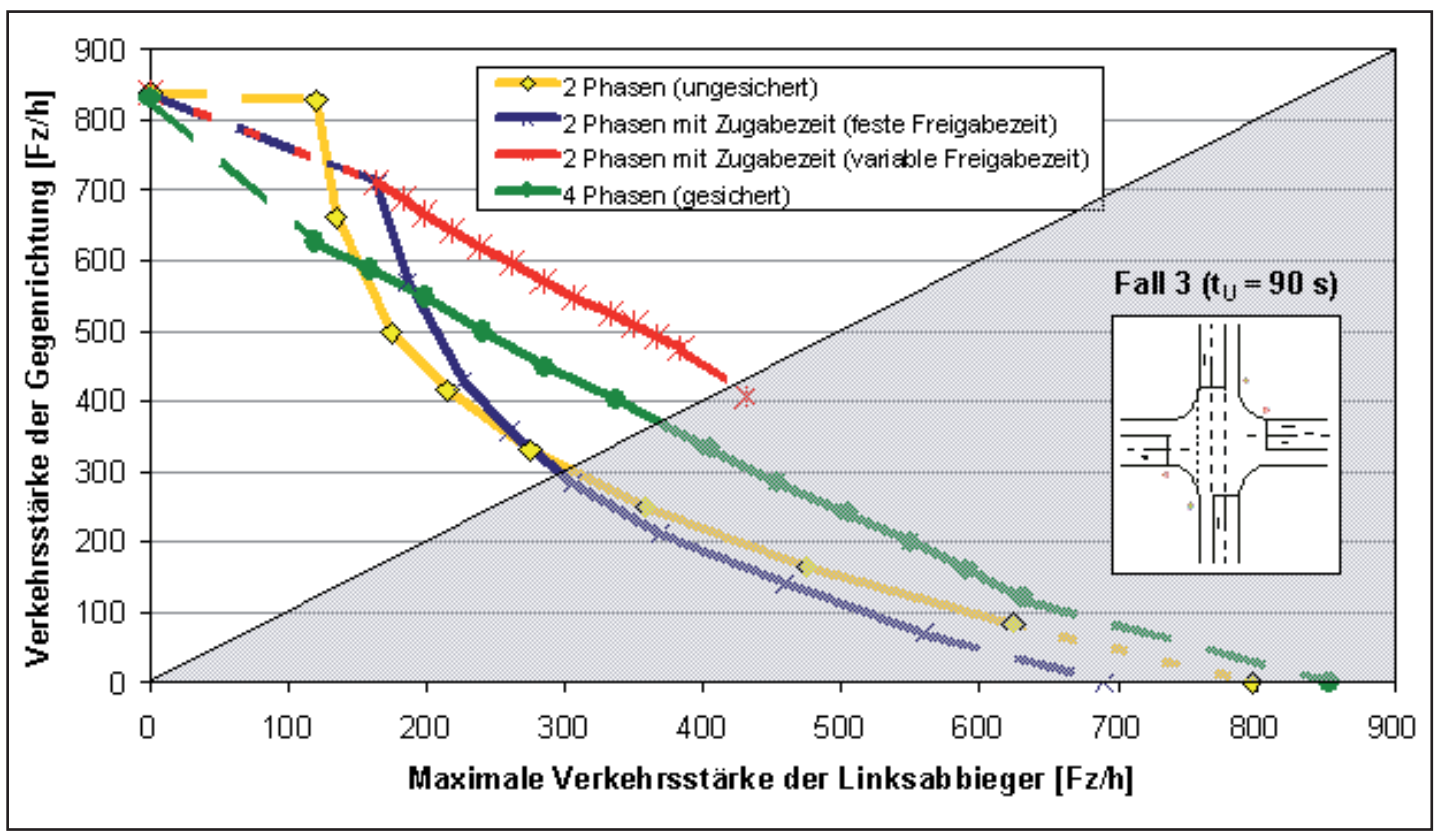

Abbildung 5:

Vergleich des Einflusses eines konfligierenden Linksabbiegers bei unterschiedlichen Steuerungsverfahren für den Linksabbiegeverkehr auf die maximale Verkehrsstärke der übergeordneten Gegenrichtung für mittlere Kreuzungen für eine Umlaufzeit von 90 s

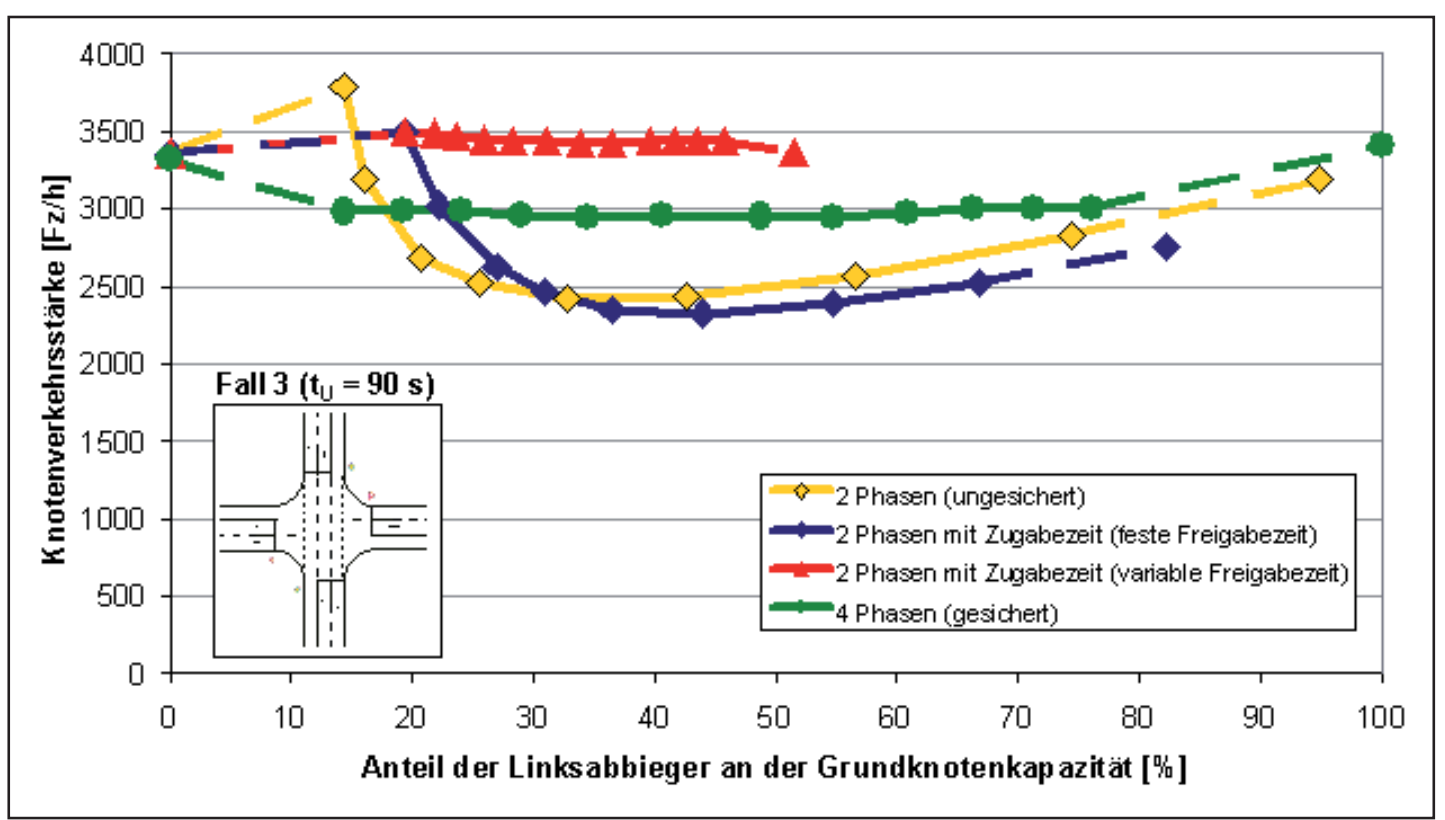

Abbildung 6:

Knotenverkehrsstärke bei verschiedenen Steuerungsformen für mittlere Kreuzungen für eine Umlaufzeit von $90 \mathrm{~s}$ 
- Die höchsten Leistungsfähigkeitsergebnisse erzielt eine Steuerung bei variabler Zugabezeit. Die Gesamtleistungsfähigkeit liegt bei ca. $3500 \mathrm{Fz} / \mathrm{h}$. Diese Lösung kommt allerdings nur bei unsymmetrischer Belastung infrage, da sie jeweils nur für eine Richtung vorgesehen werden kann.

Insgesamt zeigt sich also, dass bei größeren Linksabbiegeverkehren die gesicherte Freigabe immer auch unter Leistungsfähigkeitsgesichtspunkten günstigere Werte ergibt als das Zweiphasensystem. Der Anteil der Linksabbieger ist allerdings etwas höher als bei mehrstreifigen Knotenpunktzufahrten, da dort die Verkehrsstärke der Geradeausströme wesentlich größere Werte erreichen kann. Die Absolutgröße der kritischen Linksabbiegeströme liegt in beiden Fällen bei etwa $500 \mathrm{Fz} / \mathrm{h}$ in allen Zufahrten insgesamt.

\subsubsection{Kleine Kreuzung mit je einem Fahr- streifen in allen Zufahrten (Fall 1)}

Diese Geometrie führt zur Nutzung der Fahrstreifen als Mischfahrstreifen für alle Verkehrsbeziehungen. Dies führt zu einem völlig anderen Verkehrsablauf als in den vorher betrachteten Fällen, da der Linksabbieger nicht gesondert von den anderen Verkehrsströmen fließen kann bzw. alle anderen Verkehrsströme ebenfalls von den Linksabbiegern in der gleichen Richtung beeinflusst werden. Die bisher verwendete Simulationsstrategie der Vollauslastung führt nur eingeschränkt zu aussagefähigen Ergebnissen, da die Linksabbieger keine Restriktionen erfahren und daher die Verkehrsbeziehungen sich ohne weitere Randbedingungen nahezu gleich verteilt herausbilden. Es wurde deshalb eine zweite Simulationsserie gerechnet, bei der die Verkehrsstärke der Linksabbieger im Rahmen eines Anteils von $10 \%$ der Gesamtverkehrsstärke jeder Zufahrt begrenzt wurde.

Bei einer Verkehrsstärke der Linksabbieger und der Gegenrichtung für beide Lastfälle (10\% und $33 \%$ ) ergibt sich Folgendes:

- Bei der Annahme des Fehlens von Linksabbiegern stellt sich eine maximale Verkehrsstärke von $800 \mathrm{Fz} /$ Richtung ein.

- Bei bereits geringen Verkehrsstärken der Linksabbieger tritt ein erheblicher Rückgang der Verkehrsstärke in der Gegenrichtung auf, gleichgültig ob nur zwei Phasen geschaltet werden oder ob Zugabezeiten in fester oder variabler Länge hinzukommen.

- Verringert man die Verkehrsstärke der Linksabbieger bei gleichbleibendem Anteil an der Verkehrsstärke, so gehen ebenfalls die Fahrzeugmengen in der Gegenrichtung zurück. Lässt man mehr Linksabbieger zu und erhöht den Anteil bis auf $33 \%$, vermindert sich notwendigerweise ebenfalls die Verkehrsstärke der Gegenrichtung.

- Eine gesicherte Freigabe ist nur in Form der Freigabe einzelner Zufahrten möglich.

Für die Gesamtleistungsfähigkeit des Knotenpunktes bedeutet dies, dass ohne Linksabbieger ca. $3200 \mathrm{Fz} / \mathrm{h}$ den Knoten passieren können. Dieser Wert verringert sich bereits bei kleinen Linksabbiegeanteilen ohne gesicherte Freigabe oder bei einer festen bzw. variablen Zugabezeit deutlich auf Werte unter zwei Drittel des Ausgangsniveaus.

Bei der Zufahrtsignalisierung können $1200 \mathrm{Fz} / \mathrm{h}$ den Knoten befahren. Das ergibt sich aus dem 4fachen der einzelnen Zufahrtverkehrsstärken. Dieser Wert bleibt konstant ohne Einfluss des Linksabbiegeanteils.

Insgesamt ist für derartige Geometrien festzuhalten, dass sich nur sehr ungünstige Leistungsfähigkeiten unter Berücksichtigung des Linksabbiegeverkehrs ergeben können. Es sollte daher unbedingt angestrebt werden, zumindest in den einzelnen Zufahrten nach Möglichkeit Linksabbiegestreifen zu schaffen. 


\subsubsection{Kombination ungleicher Knoten- punktzufahrten an Kreuzungen}

In der Realität werden sehr häufig unterschiedliche Zufahrten an Kreuzungen miteinander verknüpft. In diesen Fällen können mithilfe der jeweiligen Darstellung von Verkehrsstärken an den Konfliktflächen die dort sich einstellenden maximalen Leistungsfähigkeiten aus Kombination der unterschiedlichen Verhältnisse zusammengesetzt werden. In Tabelle 1 sind alle theoretisch möglichen Kombinationen der vier untersuchten unterschiedlichen Zufahrten dargestellt. Die untersuchten Kombinationen sind farbig hinterlegt. Weitere sinnvolle Kombinationen sind gekennzeichnet.

Im Ergebnis stellen sich dann Werte ein, die jeweils zwischen den betrachteten Gesamtsituationen einheitlicher Bauart liegen. Diese Kombinationen wurden ohne zusätzliche Simulationsrechnungen aus den vorhandenen Ergebnissen in Analogieschlüssen abgeleitet, d. h., es wurden keine neuen Zwischenzeiten in Ansatz gebracht. Die Ergebnisse zeigen, dass die Leistungsfähigkeitsunterschiede zwischen gesicherten und ungesicherten Steuerungen im Bereich mittlerer bis hoher Linksabbiegeströme bestätigt werden. Meist hat die gesicherte Steuerungsvariante erhebliche Vorteile für die Gesamtknotenkapazität, in keinem Fall - mit Aus- nahmekleiner Linksabbiegeanteile(biszu10 \%)ist die 2-Phasensteuerung leistungsfähiger.

\subsubsection{Gesicherte Freigabe des Linksabbie- gers als Vorgabezeit, Zugabezeit oder kombiniert, jeweils mit fester und va riabler Länge}

Aus Gründen der Verkehrssicherheit wird in aller Regel bei einer Verlängerung der Freigabezeit zugunsten der Linksabbieger eine Zugabezeit gewählt, d. h., im Anschluss an die Freigabezeit des Gegenverkehrs wird dieser angehalten und dem Linksabbieger wird eine „Zugabezeit“ gegeben. Wird die zusätzliche Freigabezeit vor Beginn der Freigabe des Gegenverkehrs eingerichtet, so entsteht eine Vorgabezeit. Für Fall 3 und eine Umlaufzeit von $90 \mathrm{~s}$ wurde die unterschiedliche Auswirkung verschiedener Vor- und Zugabezeitvarianten überprüft. Dabei zeigt sich:

- Vorgabezeiten erreichen etwas höhere Leistungsfähigkeiten als Zugabezeiten.

- Die Regelung mit Vorgabezeit oder Zugabezeit ist jeweils nur in einer Fahrtrichtung möglich. Die Gegenrichtung muss jeweils ohne eigene Freigabezeit für den Linksabbieger auskommen. Dies ist also nur sinnvoll bei unsymmetrischer Verkehrsbelastung.

- Ein Angebot für beide Fahrtrichtungen führt notwendigerweise zu einer Kombination von Vor- und Zugabezeiten. Dies erlaubt bei

Tabelle 1:

Kategorisierung der Knotenpunktformen

\begin{tabular}{|c|c|c|c|c|c|c|c|c|}
\hline \multicolumn{5}{|c|}{ Hauptrichtung } & \multirow{2}{*}{\multicolumn{4}{|c|}{$\begin{array}{l}\text { Nebenrichtung } \\
\text { Knotenpunktform }\end{array}$}} \\
\hline \multirow{2}{*}{$\begin{array}{l}\text { KP- } \\
\text { Form }\end{array}$} & \multicolumn{3}{|c|}{ Anzahl Fahrstreifen } & \multirow{2}{*}{$\begin{array}{l}\text { Dreiecks- } \\
\text { insel }\end{array}$} & & & & \\
\hline & LA & GE & RA & & Fall 1 & Fall 3 & Fall 4 & Fall 6 \\
\hline Fall 1 & \multicolumn{3}{|c|}{1} & nein & + & - & - & - \\
\hline Fall 3 & 1 & \multicolumn{2}{|c|}{1} & nein & + & + & - & - \\
\hline Fall 4 & 1 & 1 & 1 & nein & - & + & + & - \\
\hline Fall 6 & 1 & 1 & 1 & ja & - & + & + & + \\
\hline \multicolumn{4}{|c|}{ Anmerkung: } & - $\quad$ nic & sinnvoll & \multicolumn{2}{|c|}{ untersucht } & nbiniert \\
\hline
\end{tabular}


höheren Verkehrsstärken der Gegenrichtung eine größere Verkehrsmenge der Linksabbieger. Zu berücksichtigen sind aber schwer verständliche Signalzustände.

- Variable Vorgabezeiten oder Zugabezeiten können nur begrenzte Längen erhalten.

\subsubsection{Auswirkungen unterschiedlicher Umlaufzeiten}

Generell wirken sich kürzere Umlaufzeiten derart aus, dass die Verluste bei Phasenwechseln anteilig größer werden, weil die Zwischenzeiten absolut gleiche Länge erhalten müssen. Dies führt generell zu einer Zunahme der Knotenpunktkapazität bei steigender Umlaufzeit. Allerdings erfolgt die Zunahme der Kapazität nicht linear mit der Umlaufzeit, weil auch die Verlustzeiten anteilig bei größeren Umlaufzeiten kleinere Veränderungen bewirken.

\subsubsection{Mögliche Verkehrsstärken von Links- abbiegern bei hoher Belastung im Gegenverkehr}

Bei allen mehrstreifigen Varianten von Knotenpunktzufahrten tritt bei geringen Linksabbiegeverkehrsstärken der Fall auf, dass bis zu ca. 120 Linksabbieger/h (bei einer Umlaufzeit von 90 s) während der Phasenwechsel abfließen können und daher auch bei ungesicherter Freigabe keine Einschränkungen des Geradeausverkehrs auftreten. Bis zu dieser Belastung hat eine gesicherte Freigabe grundsätzlich Leistungsfähigkeitseinbußen für den Gegenverkehr zur Folge, weil die Zeit für die Sonderphase dem Gegenverkehr entzogen wird. Je mehr Umläufe in einer Stunde auftreten (bei kürzeren Umlaufzeiten), desto mehr Linksabbieger können während der Phasenwechsel abgefertigt werden. Andererseits ist die Zahl aber abhängig von der Knotenpunktgeometrie (Anzahl der Aufstellplätze im Knoten).
Der kapazitätsmindernde Effekt der Sonderphase im Bereich kleiner Linksabbiegeströme ist geringer, wenn Zugabezeiten eingerichtet werden. Dies ist aber nur möglich in jeweils einer Fahrtrichtung (unsymmetrische Lösung).

Sobald mehr als 120 Fahrzeuge als Linksabbieger auftreten, haben gesicherte Freigaben für diese Ströme teilweise deutlich höhere Leistungsfähigkeiten als die Zweiphasigkeit. Die Einrichtung einer Zugabezeit (die unsymmetrische Belastungsverhältnisse voraussetzt) erreicht immer bessere Werte, die in den Bereich höherer Verkehrsstärken von Linksabbiegern verschoben werden können durch variable Freigabezeiten. Treten starke Linksabbiegeströme in beiden Fahrtrichtungen auf, so muss in aller Regel eine 4-Phasensteuerung gewählt werden, wenn Vorgabezeiten vermieden werden sollen (weil sie Sicherheitsnachteile haben).

Die maximal mögliche Verkehrsstärke für Linksabbieger in Abhängigkeit des Steuerungsverfahrens bei unterschiedlichen geometrischen Ausgangssituationen zeigt Abbildung 7. Die dort dargestellten Werte sind aus den Diagrammen abgelesen und auf praktische Werte gerundet worden. Es wird von einer Fahrstreifenbelastung in Höhe von $600 \mathrm{Fz} / \mathrm{h}$ ausgegangen (bei zwei Fahrstreifen in der Gegenrichtung, also 1200 Fz/h). Es zeigt sich, dass bei 2-Phasensteuerung nur unwesentlich mehr Fahrzeuge als während des Phasenwechsels abfließen können. Bei gesicherter Freigabe ist dieser Wert teilweise erheblich größer, d. h., insbesondere in den Fällen mit mehreren Fahrstreifen kann in der Sonderphase durch größere Zeitanteile eine erhebliche Verkehrsleistung des Linksabbiegers erreicht werden. Die variable Zugabezeit erreicht den höchsten Abfluss der Linksabbieger, kann aber nur jeweils für eine Fahrtrichtung realisiert werden.

Eine besondere Rolle spielt der kleine Knotenpunkt mit nur einem Fahrstreifen je Zufahrt: Die 
Ergebnisse hier sind nicht vergleichbar mit den anderen Kreuzungen. Die Freigabe in gesicherten Phasen führt in diesen Fällen zu einer spürbaren Reduktion der Gesamtleistungsfähigkeit auf der Konfliktfläche. 300 Fahrzeuge können diese Konfliktfläche je Stunde befahren (dies ist die Kapazität der einstreifigen Knotenpunktzufahrt bei Zufahrtsignalisierung). Je größer die Linksabbiegeverkehrsstärke wird, desto kleiner ist die Leistungsfähigkeit für den restlichen Verkehr.

\subsubsection{Gesamtkapazitäten von Knotenpunk- en mit unterschiedlicher Steuerungs- form}

Die Gesamtkapazität am Knotenpunkt erreicht ca. $3300 \mathrm{Fz} / \mathrm{h}$ bei zweistreifigen Zufahrten und etwas über 6000 Fahrzeuge bei dreistreifigen Zufahrten. Wenn Rechtsabbieger ungehindert abfließen können (neben nicht signalisierten Dreiecksinseln) erhöht sich der Wert noch auf nahezu $7000 \mathrm{Fz} / \mathrm{h}$.

Sobald Linksabbieger in nennenswerter Zahl auftreten, wird die Gesamtkapazität des Kno- tenpunktes vor allem im Fall ungesicherter Freigabe bei ca. 15 \%-Anteil durch Linksabbieger (zweistreifige Zufahrten) oder $7 \%$ (dreistreifige Zufahrten) vermindert. Die Mehrphasensysteme zeigen dagegen nur einen geringen Rückgang der Knotenpunktkapazität bei Ansteigen des Linksabbiegeanteils. Unter günstigen Verhältnissen bleibt die Gesamtkapazität nahezu konstant. Die Sicherung des Linksabbiegers durch eine Zugabezeit, insbesondere wenn diese variabel gehalten werden kann, bringt mindestens bis in den Bereich von 20 \% Linksabbiegeanteil teilweise noch deutlich höhere Gesamtkapazitäten als bei 4-Phasensteuerung.

Ein weiterer Sonderfall ist die große Kreuzung mit freien Rechtsabbiegern (Fall 6): Hier gewinnt ausschließlich die Sicherung mit Zugabezeiten an Kapazität gegenüber den ungesicherten Zweiphasensystemen, während die Mehrphasensysteme maximal gleiche Kapazitäten im Bereich mittlerer Linksabbiegeanteile erreichen können. Dies liegt daran, dass bei ungesicherten Zweiphasensystemen die Rechtsabbieger kontinuierlich frei fließen können. Der Effekt tritt also

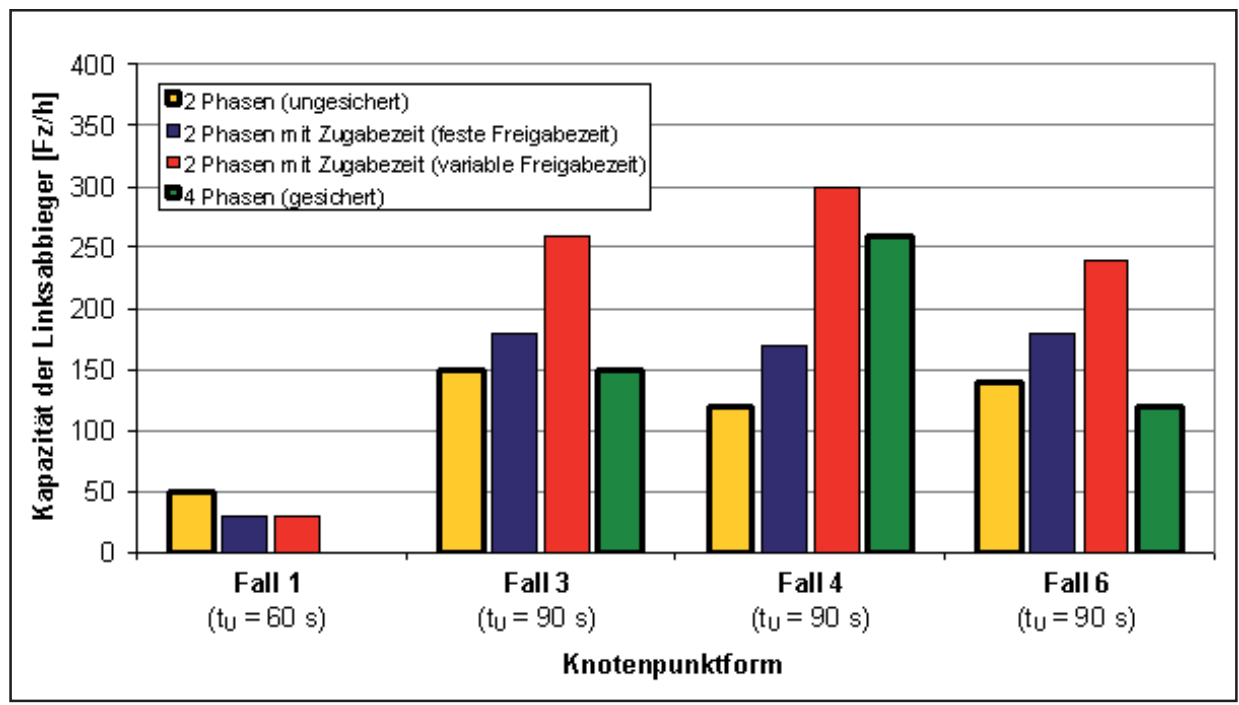

Abbildung 7:

Kapazitäten der Linksabbieger bei einer Gegenverkehrsstärke von 600 Fz/(h*Fahrstreifen) für unterschiedliche Steuerungsvarianten der Linksabbieger und Knotenpunktformen ein Ergebnis der Simulationsläufe 
nur ein, wenn auch hoch belastete Rechtsabbiegerströme vorhanden sind. Dann wird am Umkehrschluss aber auch ein großer Linksabbiegeanteil auftreten, so dass die Werte der geringen Linksabbiegebelastung nicht realistisch sein werden.

\section{Einsatzempfehlungen}

Aufgrund der höheren Sicherheit mehrphasiger Systeme sollten generell keine ungesicherten Zweiphasensysteme mehr zum Einsatz kommen. Aus verkehrstechnischen Gründen ergibt sich in einzelnen Fällen eine Einschränkung der Leistungsfähigkeit durch die zusätzliche Linksabbiegesicherung. Es werden folgende Einsatzempfehlungen gegeben:

(1) Bei hohen Verkehrsbelastungen, z. B. bei Knotenverkehrsstärken von mehr als 6500 Kfz-Überfahrten/h müssen mehrere Fahrstreifen für einzelne Fahrbeziehungen angelegt werden. Dann kommt eine ungesicherte Freigabe des Linksabbiegers generell nicht infrage, weil die Sicherheit nicht zu gewährleisten ist.

(2) Liegt die Verkehrsstärke zwischen 4600 und $6500 \mathrm{Fz} / \mathrm{h}$, so sind meist aus allen Richtungen dreistreifige Knotenpunktzufahrten erforderlich. Gleichmäßige Auslastung der Verkehrsbeziehungen vorausgesetzt, ergibt sich ein mittlerer Linksabbiegeanteil um $25 \%$.

- Wird der Rechtsabbieger neben Dreiecksinseln zeitweise frei geführt, so erreicht die 4-Phasensteuerung gleiche $\mathrm{Ka}$ pazitätswerte wie 2-Phasensteuerungen. Die Sicherung der Linksabbieger mit einer Zugabezeit wird in aller Regel nicht mehr funktionsfähig sein, da sie eine Konzentration der Linksabbieger auf maximal zwei der vier Fahrtrichtungen erfordert (unsymmetrische Belastungszustände). Aus Sicherheitsgründen soll die Möglich- keit des Durchsetzens nicht vorgesehen werden.

- Bei dreistreifigen Zufahrten ohne Dreiecksinseln ist die 4-Phasensteuerung bei gleichmäßiger Auslastung des Knotenpunktes auch unter verkehrstechnischen Gesichtspunkten ebenso wie unter Sicherheitsgesichtspunkten die bei weitem günstigste Lösung.

(3) Bei Gesamtbelastungen von 3000 bis 4600 Fahrzeugen in der Spitzenstunde werden Mischformen von geometrischen Knotenpunktgestaltungen auftreten. Z. B. wird in der Hauptrichtung eine Aufteilung der Fahrstreifen ähnlich Fall 4 (drei Fahrstreifen ohne Dreiecksinsel) und in der Nebenrichtung zweistreifige Zufahrten entsprechend Fall 3 auftreten. Bei gleichmäßiger Auslastung, d. h. Linksabbiegeanteilen um 25 \% der Gesamtkapazität, wird auch in diesen Fällen die vierphasige Steuerung verkehrstechnisch optimale Verhältnisse bieten. Möglicherweise sind hier auch Zugabezeiten alternativ zu prüfen, solange das Durchsetzen der zweistreifigen Gegenverkehre vermieden werden kann.

(4) Für Belastungen zwischen 2000 und 3000 Fahrzeugen in der Spitzenstunde sollte bei kleinen Linksabbiegeanteilen unter $20 \%$ wie Einrichtung von Zugabezeiten geprüft werden. Bei größeren Linksabbiegeanteilen ist die 4-Phasensteuerung in jedem Fall von Vorteil.

(5) Belastungen zwischen 1000 und 3000 Fahrzeugen in der Spitzenstunde sollten keinesfalls mit einstreifigen Zufahrten geplan werden. Eine Lichtsignalsteuerung wäre dann nur mit zweiphasigem System ausreichend leistungsfähig. Die Einrichtung eines Abbiegefahrstreifens für Linksabbieger ist unbedingt zu fordern (aus Wirtschaftlichkeitsgründen auch zu vertreten).

(6) Bis zu 1200 Fahrzeugen in der Spitzenstun- 
de lassen sich einstreifige Zufahrten als Zufahrtsignalisierung mit gesicherter Freigabe der Linksabbieger einrichten. Empfohlen wird der Einsatz bis zu ca. 1000 Fz/h.

\section{$4 \quad$ Fazit}

Die Ergebnisse der Untersuchung durch die Technische Universität Dresden lassen sich wie folgt zusammenfassen:

- Bei großen Kreuzungen mit viel Verkehr ist immer ein eigenes Signal für Linksabbieger vorzusehen. Dadurch wird die Verkehrssicherheit für Abbieger, entgegenkommende Fahrzeuge, Fußgänger, sowie Radfahrer erheblich verbessert.

- Bei großen Kreuzungen mit mittlerer Belastung und kleineren Kreuzungen mit hoher Belastung ist das „gesicherte“ Linksabbiegen hinsichtlich Verkehrsablauf und Verkehrssicherheit sogar die günstigste Lösung.

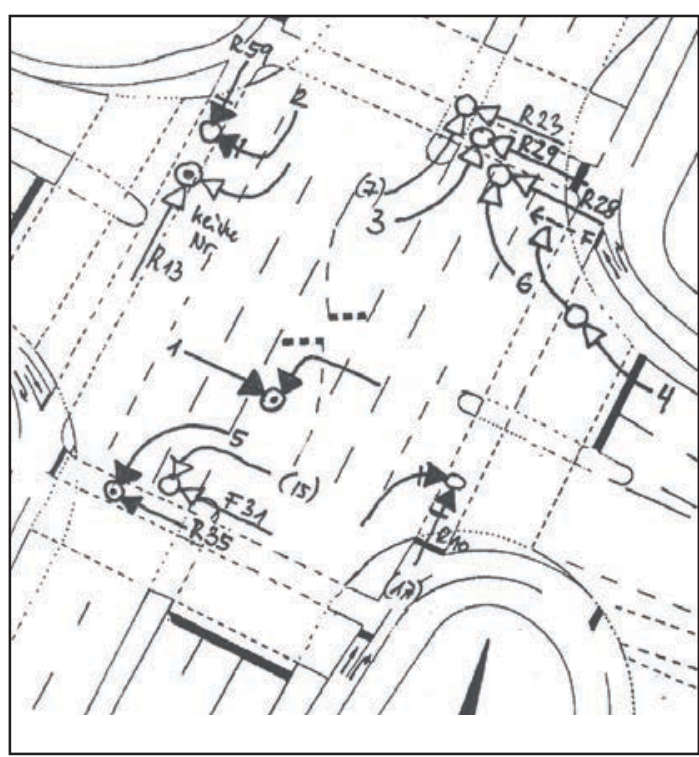

Abbildung 8:

Beispiel einer Unfallhäufung durch fehlende Phase für Linksabieger (Linksabbieger und parallel freigegebener Rad- und Fußgängerverkehr)
- Kein eigenes Linksabbiegesignal ist nur an kleinen Kreuzungen mit fehlender Abbiegespur und geringem Verkehr vertretbar.

- Unter Umständen muss das Linksabbiegen auch ganz unterbunden werden.

- Die Kosten für die Umrüstung der Ampelanlage auf eine separate Linksabbiegephase liegen in der Regel deutlich unter den vermeidbaren Unfallkosten.

\section{Auf Grundlage der Forschungsergebnisse for- dert daher die Unfallforschung der Versicherer:}

Neu angelegte Lichtsignalanlagen müssen an Kreuzungen immer eine separate Grünphase für Linksabbieger haben, bestehende Anlagen müssen bei bekannten Problemen unverzüglich umgerüstet werden, alle anderen spätestens dann, wenn ohnehin Baumaßnahmen anstehen.

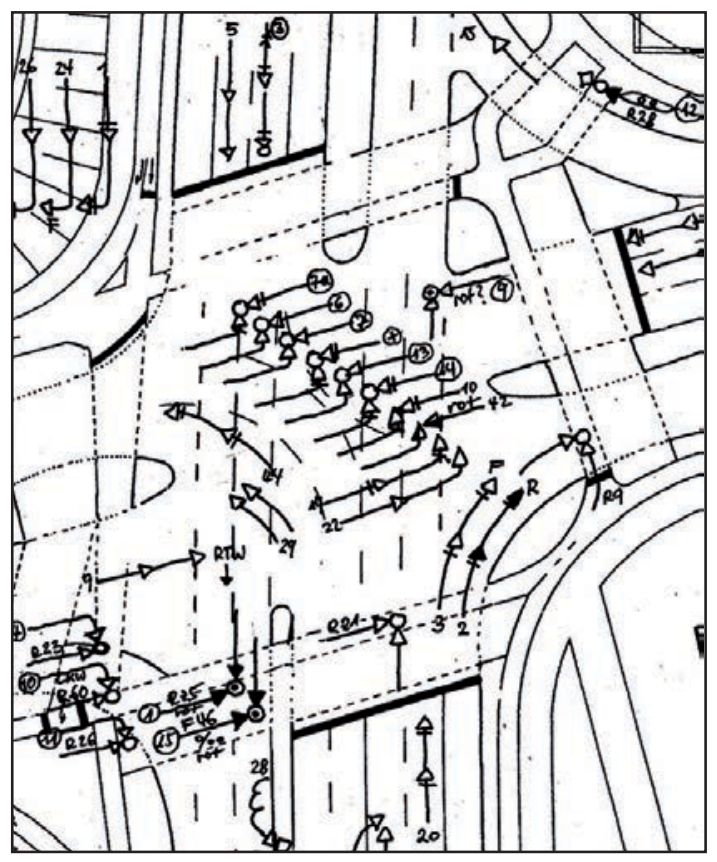

Abbildung 9:

Beispiel einer Unfallhäufung durch fehlende Phase für Linksabieger (Linksabbieger mit Gegenverkehr) 


\section{Literatur}

Forschungsgesellschaft für Straßen- und Verkehrswesen (FGSV). (1992). Richtlinien für Lichtsignalanlagen (RiLSA). Köln. Herausgabe: Arbeitsgruppe Verkehrsführung und Verkehrssicherheit. Forschungsgesellschaft für Straßen- und Verkehrswesen.

Forschungsgesellschaft für Straßen- und Verkehrswesen (FGSV). (2010). Richtlinien für Lichtsignalanlagen (RiLSA). Köln. Herausgabe: Arbeitsgruppe Verkehrsführung und Verkehrssicherheit. Forschungsgesellschaft für StraBen- und Verkehrswesen.

Allgemeine Verwaltungsvorschrift zur Straßenverkehrs-Ordnung (VwV-StVO) in der Fassung vom 26. Januar 2001 (BAnz. S. 1419, 5206) Zuletzt geändert am 2009-07-17 (BAnz. Nr. 110 vom 2009-07-29, S. 598) mWv 2009-09-01.

Scholz, Th. \& Ortlepp, J. (2010). Verkehrstechnische Auswirkungen der Sonderphase für Linksabbieger an Knotenpunkten mit Lichtsignalanlage. Erstellt durch Technische Universität Dresden, Fakultät Verkehrswissenschaften, Dresden. Herausgabe: Unfallforschung der Versicherer (UDV) im Gesamtverband der Deutschen Versicherungswirtschaft e. V. Berlin. Download unter www.udv.de

Eckstein K. \& Meewes, V. (2002). Sicherheit von Landstraßen-Knotenpunkten. Mitteilungen des Institutes für Straßenverkehr Köln (ISK). Gesamtverband der Deutschen Versicherungswirtschaft e. V. Köln 


\section{GDV}

DIE DEUTSCHEN VERSICHERER

\section{Gesamtverband der Deutschen Versicherungswirtschaft e.V.}

Wilhelmstraße 43/43G, 10117 Berlin

Postfach 0802 64, 10002 Berlin

Tel.: 030/2020 - 50 00, Fax: 030/2020-6000

www.gdv.de, www.udv.de 